\title{
COMPARISON OF PIXEL-BASED AND FEATURE-BASED APPROACH FOR SMALL OBJECT CHANGE DETECTION
}

\author{
J. Seo ${ }^{1}$, T. Kim ${ }^{1,2 *}$ \\ ${ }^{1}$ Program in Smart City Engineering, Inha University, Incheon, Republic of Korea \\ 2 Dept. of Geoinformatic Engineering, Inha University, Incheon, Republic of Korea \\ 22201180@inha.edu, tezid@inha.ac.kr
}

Commission III, WG III/7

KEY WORDS: Change Detection, Small Object, MAD, Feature point, High resolution image, SIFT, SURF

\begin{abstract}
:
Satellite image resolution has evolved to daily revisit and sub-meter GSD. Main targets of previous remote sensing were forest, vegetation, damage area by disasters, land use and land cover. Developments in satellite images have brought expectations on more sophisticated and various change detection of objects. Accordingly, we focused on unsupervised change detection of small objects, such as vehicles and ships. In this paper, existing change detection methods were applied to analyze their performances for pixel-based and feature-based change of small objects. We used KOMPSAT-3A images for tests. Firstly, we applied two change detection algorithms, MAD and IR-MAD, which are most well-known pixel-based change detection algorithms, to the images. We created a change magnitude map using the change detection methods. Thresholding was applied to determine change and non-change pixels. Next, the satellite images were transformed as 8-bit images for extracting feature points. We extracted feature points using SIFT and SURF methods to analyze feature-based change detection. We assumed to remove false alarms by eliminating feature points of nonchanged objects. Therefore, we applied a feature-based matcher and matched feature points on identical image locations were eliminated. We used non-matched feature points for change/non-change analysis. We observed changes by creating a $5 \times 5$ size ROI around extracted feature points in the change/non-change map. We determined that change has occurred on feature points if the rate of change pixels with ROI was more than $50 \%$. We analyzed the performance of pixel-based and feature-based change detection using ground truths. The F1-score, AUC value, and ROC were used to compare the performance of change detection. Performance showed that feature-based approaches performed better than pixel-based approaches.
\end{abstract}

\section{INTRODUCTION}

While spatial and temporal resolution of satellite images has been improved, one could expect more sophisticated change detection with daily revisit of satellites at sub-meter GSD (ground sampling distance). Previously, change detection from satellite image has been studied mostly for wide objects such as forest (Housman et al., 2018), damage area by disaster (Sublime, Kalinicheva, 2019), land cover (Twisa, Buchroithner, 2019), etc (Ballanti et al., 2017). With the improvement of spatial and temporal resolutions, automated detection of small objects, such as small ships and vehicles, could be carried out from satellite images (Audebert et al., 2017, Eikvil et al., 2009, Wu et al., 2020, Zheng et al., 2019). Interests on small objects have been increased rapidly. The literature has shown many researches on small object detection have used AI (Artificial Intelligence) and Deep learning for detecting and recognition object in remote sensing and computer vision (Tao et al., 2019, Radovic et al., 2017, Peng et al. 2019). These works assumed the availability of image templates of the small objects of interest.

We focus on automated change detection of small objects without a priori templates. Therefore, we extracted change/non-change maps on feature points using unsupervised-based methods. The change map was extracted using the existing unsupervised change detection methods widely used in remote sensing, MAD (Multivariate Alteration Detection), and IR-MAD (Iteratively Reweighted-MAD). Feature points were extracted using SIFT (Scale Invariant Feature Transform) and SURF (Speed-Up Robust Features). We investigated whether the change map and feature points would exhibit a change magnitude of regions and location of small objects. We also investigated whether the extracted change map and feature points could be used for localization of any changed object.

We performed change detection after pre-processing the data of study areas. We created change magnitude maps using MAD and IRMAD and generated feature points using SIFT and SURF, separately. Feature points extracted from before and after images were matched to eliminate feature points of a same object. In order to filter out false matching, only the points that matched at the same location were eliminated. A constant-size ROI was created around the filtered feature points. A ROI was overlaid on the change/non-change map to observe the rate of change on feature point locations. If the number of change pixels in the ROI was more that $50 \%$, the feature point was classified as change. The feature-based change detection performance was analyzed using classified feature points and ground truths.

We confirmed that feature-based change detection provided better performance than pixel-based change detection. The feature-based methodology indicated higher values than pixelbased in accuracy, precision, F1-score and AUC (Area Under Curve).

\footnotetext{
* Corresponding author
} 


\section{DATA}

\subsection{Dataset}

We used high resolution Kompsat 3A images over 3 study areas. Since small objects are generally agile, the images with small time gap are required for change detection. Therefore, time difference of before and after images was within 10 days. The information on the images used is shown in Table 1. We applied pan-sharpening to K3A multi-spectral bands of blue, green, red and near-infrared spectral range for change detection of small objects. We prepared ground truths for performance analysis. Ground truths was selected manually over regions where small objects were changed and over regions of non-change. Extracted small objects as ground truth include ships, vehicles, buses, trucks, containers, etc.

\begin{tabular}{|c|c|c|c|}
\hline Satellite & \multicolumn{3}{|c|}{ Kompsat 3A } \\
\hline $\begin{array}{c}\text { Study } \\
\text { Area }\end{array}$ & Incheon 1 & Incheon 2 & Seoul \\
\hline $\begin{array}{c}\text { Spatial } \\
\text { Resolution }\end{array}$ & \multicolumn{3}{|c|}{$0.5 \mathrm{~m}$ (pan-sharpened) } \\
\hline \multicolumn{3}{|c|}{ Blue: $450-520 \mathrm{~nm}$} \\
Spectral \\
Resolution
\end{tabular}

Table 1. specifications of Kompsat 3A Images.
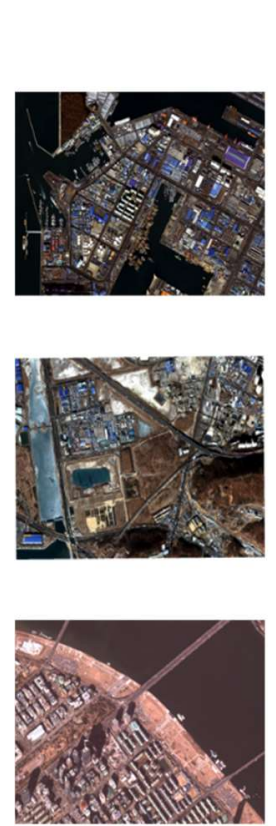

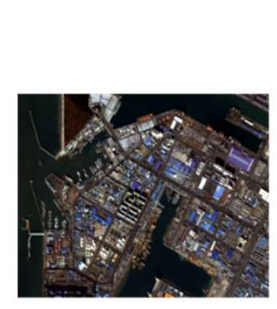

(a)

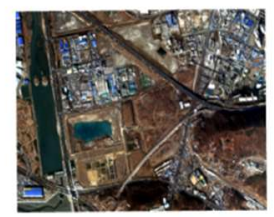

(b)

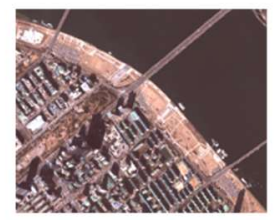

(c)
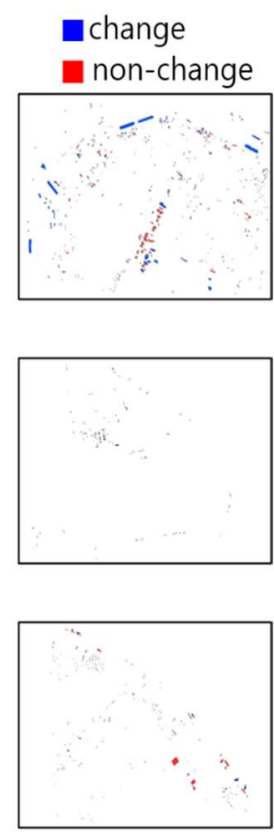

Figure 1. Our dataset of before, after image and Ground Truth over study areas ((a) Incheon 1, (b)Incheon 2, (c) Seoul)

\section{METHODOLOGY}

Our workflow of pixel-based and feature-based change detection is shown in Figure 2. The pixel-based approach includes the processes on the left-side branch of the processing tree in the figure 2. The feature-based approach includes the processes on the right-side. For the pixel-based approach, a change/non- change map was created by applying thresholding to change magnitude maps. We analyzed performance of the pixel-based approach after thresholding. For feature-based approach, we performed change magnitude calculation and feature points extraction. We re-used the change/non-change maps generated from the pixel-based approach in the feature-based approach. We created constant-size ROIs around feature points on change/nonchange maps. Within a ROI, the ratio of the change pixels was calculated. A feature point was determined as change if the ratio of change pixels was more than $50 \%$. Performance analysis was performed using ground truths. F1-score, AUC (Area Under Curve) and ROC (Receiver Operating Characteristic) were used for comparison performance.

\subsection{Pre-processing}

Earth observation data acquired from satellites need to be preprocessed. It is expected that pre-processing of remote sensing images, such as geometric correction, ortho-rectification, radiometric correction, can improve the accuracy of change detection. Therefore, we applied the geometric correction and ortho-rectification to K3A images that mainly applied in the field of change detection. Relative radiometric correction was not applied since the change detection methods we used applied relative radiometric transformation internally.

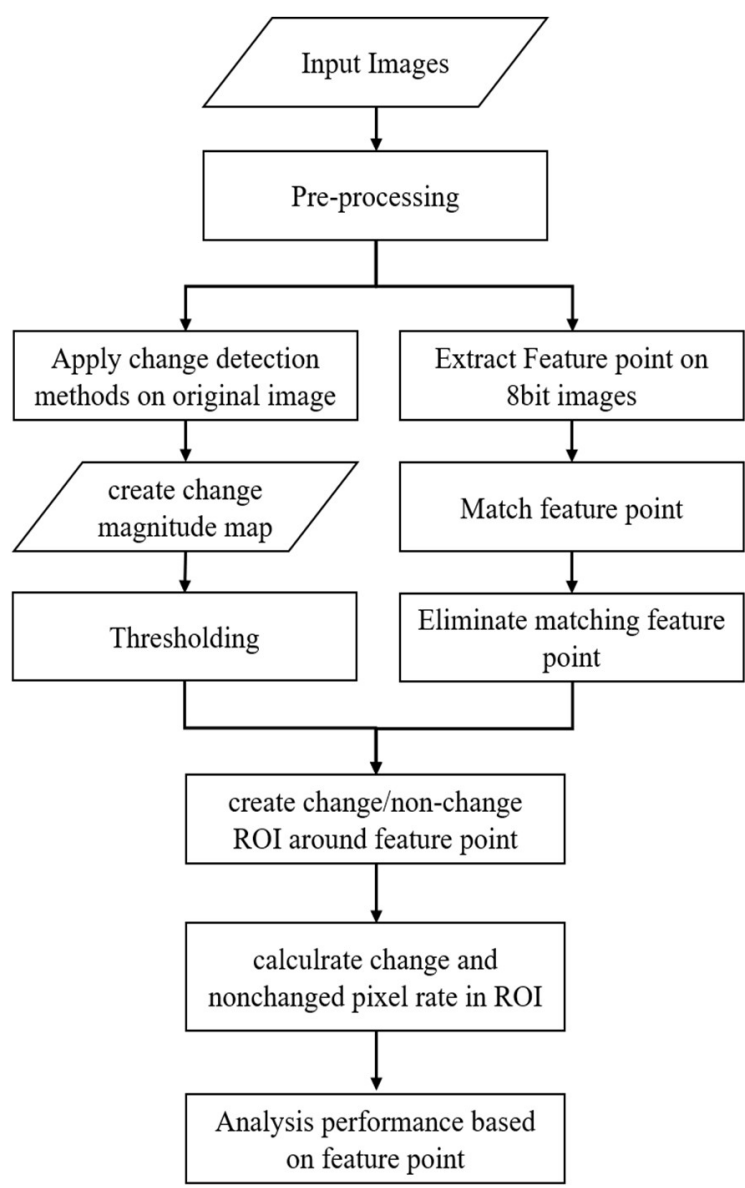

Figure 2. Our feature-based change detection workflow

\subsection{Create change/non-change map}

We calculated change magnitude maps using existing pixelbased change detection methods widely used in remote sensing community: MAD, and IR-MAD. MAD was based on canonical correlation analysis, the MAD variates are invariant to affine 
transformations. (Nielsen, 2007) The MAD has the advantage of not being sensitive to the gain and offset of the sensor and linear radiometric and atmospheric correction. IR-MAD extends MAD methods by performing MAD iteratively with reweighting. Results of change magnitude maps created by MAD and IRMAD are shown in Figure 3.

The results of MAD and IR-MAD were used for creating change/non-change maps. Change/non-change maps were created by applying thresholding to change magnitude maps. The threshold value was calculated as the minimum value of change magnitude and the standard deviation as shown in Equation 1. Change/non-change was divided using the change magnitude of the pixel position value and the threshold value as shown in Equation 2.

$$
\begin{gathered}
t h=\min +n \times s t d \\
\text { pixel }=\left\{\begin{array}{lr}
\text { change } \quad(m g>t h) \\
\text { non }- \text { change }(m g<t h)
\end{array}\right.
\end{gathered}
$$

\section{Where $\quad$ th $=$ thresholding value}

Min = minimum of change magnitude $\mathrm{n}=$ constant number std $=$ standard deviation of change magnitude $\mathrm{mg}=$ change magnitude of pixel
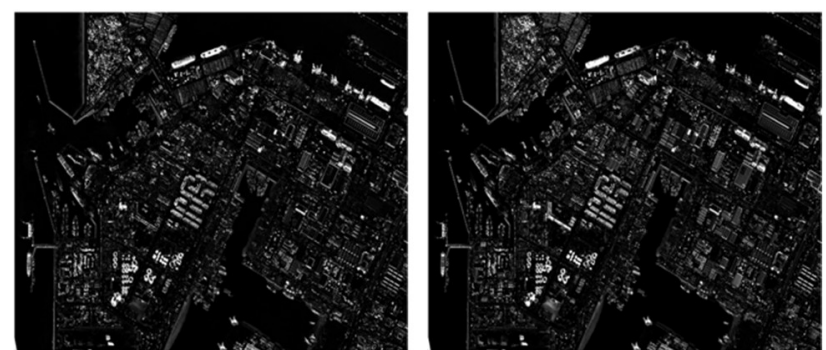

(a)
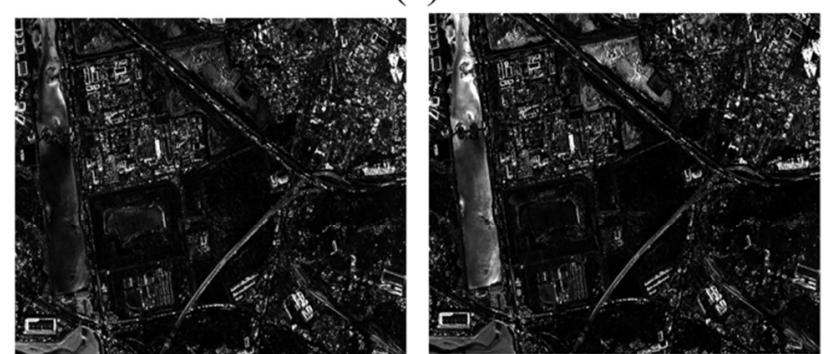

(b)
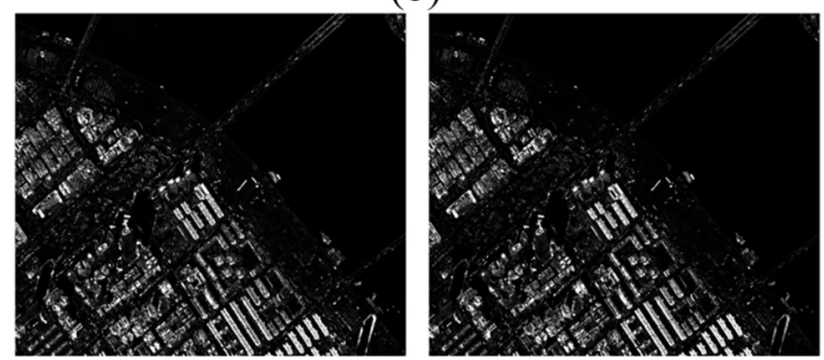

(c)

Figure 3. Result of created change magnitude map, Left: MAD, Right: IR-MAD ((a) Incheon 1, (b)Incheon 2, (c) Seoul)

\subsection{Feature-based change detection}

We transformed from 16-bit of original $\mathrm{K} 3 \mathrm{~A}$ images to 8-bit images. We extracted feature points using SIFT and SURF method from 8-bit images. We performed matching to feature points extracted from temporal images. Matching was performed on bucketed image regions by dividing the $\mathrm{K} 3 \mathrm{~A}$ images into 200x200 clipped buckets for reducing false matching (Figure 4). Feature points with a distance difference of 10 pixels or less between the two matched points were classified as non-change features as shown in Figure 5. In this way, we aimed to eliminate false alarmed feature points of non-changed objects. We could expect precise change detection by eliminating the feature points of non-change objects.
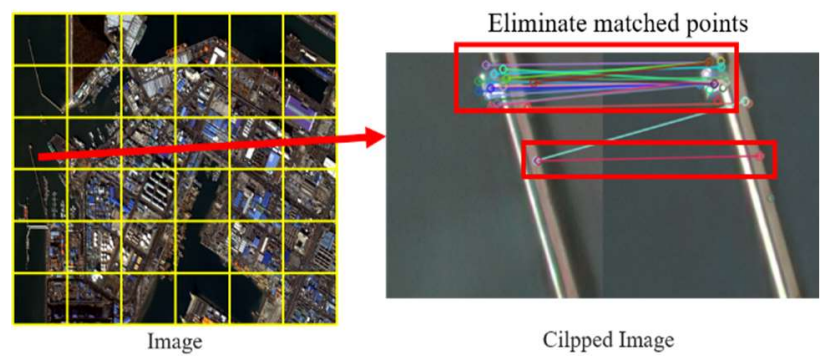

Figure 4. Eliminate matched feature points on clipped bucket

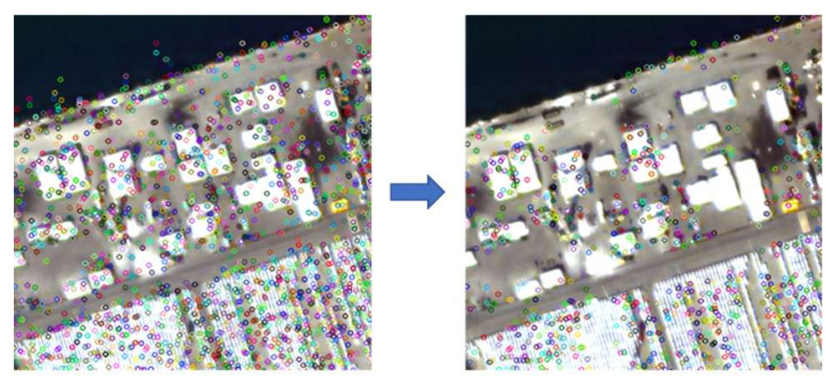

result of matching about feature point of before image

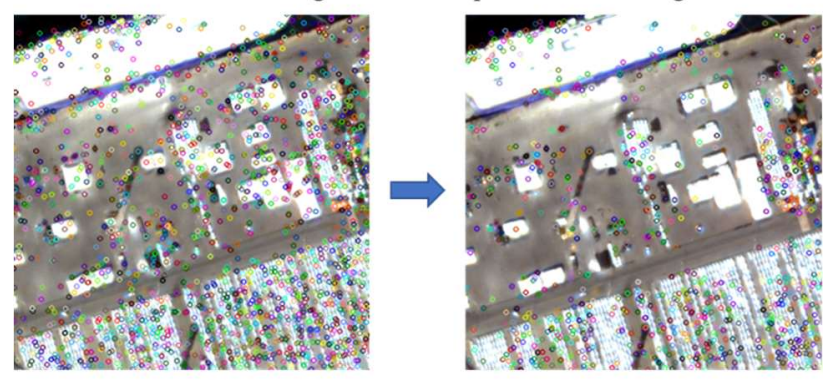

result of matching about feature point of after image

Figure 5. Result of eliminating feature point through matching

We used an ROI approach to reduce the influence of peaked pixel values at a specific pixel. We defined an ROI (Region of Interest) with the size of $5 \times 5$ pixels on extracted features. The ROI was overlaid on the change/non-change map created through Equation 2. When the number of change pixels in the ROI was checked.

We observed change magnitude around feature points. After applying a threshold to the change magnitude of feature points, we analyzed pixel-based and feature-based performance using ground truths. For performance analysis, we calculated ROC and AUC, F1-score. AUC was calculated as the area of ROC. ROC was a graph representing the relationship between false alarm and recall according to multi threshold values. The ratio of false alarm and recall ranges from 0 to 1 . A lower false alarm and 
higher recall indicates good performance. Therefore, AUC values close to 1 indicate good performance. F1-score is harmonic mean of precision and recall. We used this to verify the suitability for change detection of small objects. Recall, Precision, False alarm and F1-score were calculated as in Equation 3.

$$
\begin{gathered}
R e=\frac{T P}{T P+F N}, \quad \text { Pre }=\frac{T P}{T P+F P}, F A=\frac{F P}{F P+T N} \\
F 1-\text { score }=2 \times \frac{\text { Pre } \times \text { Re }}{\text { Pre }+ \text { Re }}
\end{gathered}
$$

Where $\quad \mathrm{TP}=$ True Positive

$\mathrm{FN}=$ False Negative

$\mathrm{TN}=$ True Negative

$\mathrm{FP}=$ False Positive

$\mathrm{Re}=$ Recall

Pre $=$ Precision

$\mathrm{FA}=$ False alarm

\section{RESULT}

The ROCs of pixel-based and feature-based change detection are shown in Figure 6. Qualitatively, performance improved when SURF feature points were used in Incheon 2 and Seoul than pixelbased change detection. The performance of feature-based approach was improved than pixel-based in all three study areas. When SIFT was used, most of the performance index represented good performance in Incheon 1. The accuracy and AUC decreased in Seoul case while the precision and F1-score improved. When SURF was used, it represented good performance in all study areas. The accuracy, precision, F1-score and AUC improved than pixel-based approaches. We found that SURF feature-based change detection provides better performance than pixel-based change detection using ROC and performance index. Results of small object change areas processed per feature are shown in Figure 7.

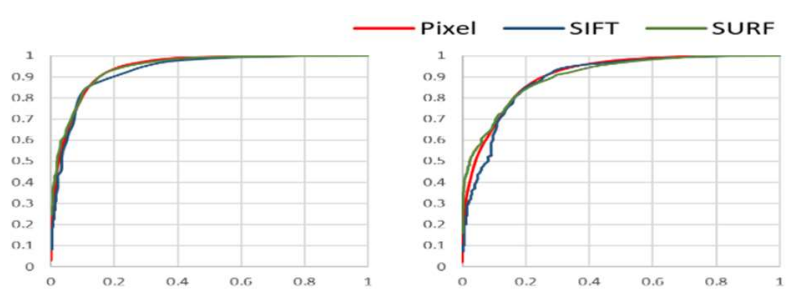

(a)
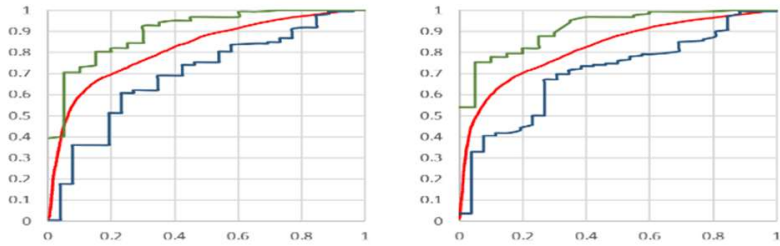

(b)
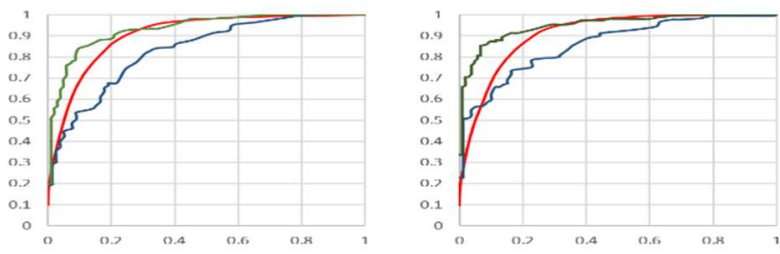

(c)

Figure 6. Comparison ROC of pixel-based and feature-based change detection methods, Left: MAD, Right: IR-MAD (((a)

\begin{tabular}{|c|c|c|c|c|c|c|c|}
\hline $\begin{array}{l}\text { Study } \\
\text { Area }\end{array}$ & Method & Domain & Accuracy & Precision & Recall & F1-score & AUC \\
\hline \multirow{6}{*}{ Incheon 1} & \multirow{3}{*}{ MAD } & Pixel & 0.8891 & 0.9035 & 0.9299 & 0.9165 & 0.9297 \\
\hline & & SIFT & 0.9216 & 0.9366 & 0.9742 & 0.9551 & 0.9180 \\
\hline & & SURF & 0.8935 & 0.8969 & 0.9639 & 0.9292 & 0.9304 \\
\hline & \multirow{3}{*}{ IR-MAD } & Pixel & 0.8462 & 0.8461 & 0.9350 & 0.8884 & 0.8983 \\
\hline & & SIFT & 0.9066 & 0.9382 & 0.9536 & 0.9458 & 0.8872 \\
\hline & & SURF & 0.8470 & 0.8550 & 0.9502 & 0.9001 & 0.8970 \\
\hline \multirow{6}{*}{ Incheon 2} & \multirow{3}{*}{ MAD } & Pixel & 0.8035 & 0.8162 & 0.9657 & 0.8847 & 0.8162 \\
\hline & & SIFT & 0.8696 & 0.8722 & 0.9937 & 0.9290 & 0.6952 \\
\hline & & SURF & 0.9085 & 0.9098 & 0.9918 & 0.9490 & 0.8996 \\
\hline & \multirow{3}{*}{ IR-MAD } & Pixel & 0.8021 & 0.8266 & 0.9446 & 0.8817 & 0.8175 \\
\hline & & SIFT & 0.8696 & 0.8722 & 0.9937 & 0.9290 & 0.6989 \\
\hline & & SURF & 0.9155 & 0.9365 & 0.9672 & 0.9516 & 0.9119 \\
\hline \multirow{6}{*}{ Seoul } & \multirow{3}{*}{ MAD } & Pixel & 0.8357 & 0.6409 & 0.7815 & 0.7042 & 0.9007 \\
\hline & & SIFT & 0.7885 & 0.7890 & 0.9503 & 0.8622 & 0.8237 \\
\hline & & SURF & 0.8577 & 0.8599 & 0.9060 & 0.8824 & 0.9278 \\
\hline & \multirow{3}{*}{ IR-MAD } & Pixel & 0.8352 & 0.6366 & 0.7954 & 0.7072 & 0.9056 \\
\hline & & SIFT & 0.8038 & 0.8250 & 0.9116 & 0.8661 & 0.8558 \\
\hline & & SURF & 0.8814 & 0.8889 & 0.9128 & 0.9007 & 0.9453 \\
\hline
\end{tabular}
Incheon 1, (b)Incheon 2, (c) Seoul)

Table 2. Performance Result of pixel-based and feature-based change detection. 

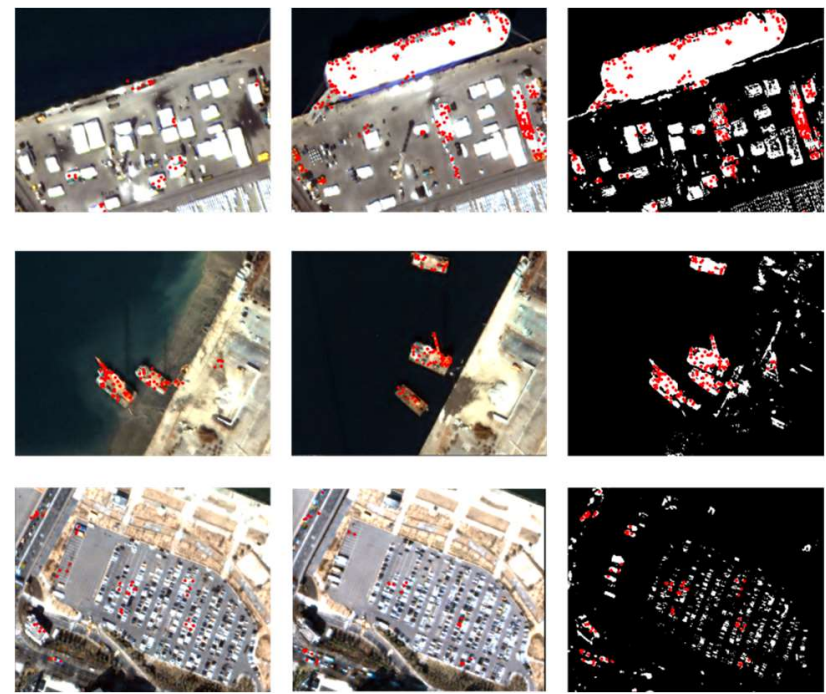

Figure 7. Result of small object change areas (red point) processed per feature. (Left: before image, Middle: after image, Right: Result image)

\section{CONCLUSION}

We could improve the change detection performance of small objects by using feature points. Among the two feature point extraction methods used, SURF outperformed. We observed that the feature-based approach based on SURF has improved change detection performance in all study areas.

There are some details that still need improvements. In the case of the small vehicles, there were undetected change objects. Changed area was too small and feature points could not be extracted. In addition, research on calculation of optimal threshold values should be conducted to perform automatic change detection.

\section{ACKNOWLEDGEMENTS}

Funds for research were provided by Satrec Initiative.

\section{REFERENCES}

Ballanti, L., Byrd, K.B., Woo, I., Ellings, C., 2017. Remote sensing for wetland mapping and historical change detection at the Nisqually River Delta. Sustainability, 9(11), 1919-1950.

Housman, I.W., Chastain, R.A., Finco, M.V., 2018. An Evaluation of Forest Health Insect and Disease Survey Data and Satellite-Based Remote Sensing Forest Change Detection Methods: Case Studies in the United States. Remote Sens., 10(8), 1184-1204.

Twisa, S., Buchroithner, M.F., 2019. Land-Use and Land-Cover (LULC) Change Detection in Wami River Basin, Tanzania. Land, 8(9), 136-150.

Sublime, J., Kalinicheva, E., 2019. Automatic Post-Disaster Damage Mapping Using Deep-Learning Techniques for Change Detection: Case Study of the Tohoku Tsunami. Remote Sens., 11(9), 1123-1142.

Peng, D., Zhang, Y., Guan, H., 2019. End-to-end change detection for high resolution satellite images using improved unet++. Remote Sens., 11(11), 1382-1404.
Audebert, N., Saux, B. L. and Lefèvre, S., 2017. Segment-beforeDetect: Vehicle Detection and Classification through Semantic Segmentation of Aerial Images. Remote Sens., 9(4), 368-385.

Eikvil, L., Aurdal, L. and Koren, H., 2009. Classification-based vehicle detection in high-resolution satellite images. ISPRS Journal of Photogrammetry and Remote Sensing, 64(1), 65-72.

Wu, X., Li, W., Hong, D., Tian, J., Tao, R. and Du, Q., 2020. Vehicle detection of multi-source remote sensing data using active finetuning network. ISPRS Journal of Photogrammetry and Remote Sensing, 167, 39-53.

Zheng, K., Wei, M., Sun, G., Anas B. and Li, Y., 2019. Using Vehicle Synthesis Generative Adversarial Networks to Improve Vehicle Detection in Remote Sensing Images. ISPRS Int. J. GeoInf., 8(9), 390-403

Nielsen, A. A. 2007. The regularized iteratively reweighted MAD method for change detection in multi-and hyperspectral data. IEEE Transactions on Image processing, 16(2), 463-478.

Radovic, M., Adarkwa, O., Wang, Q., 2017. Object Recognition in Aerial Images Using Convolutional Neural Networks. J. Imaging, 3(2), 21-29.

Tao, C., Mi, L., Li, Y., Qi, J., Xiao, Y., Zhang, J., 2019. Scene Context-Driven Vehicle Detection in High-Resolution Aerial Images. IEEE Transactions on Geoscience and Remote Sensing, 57(10), 7339-7351. 